\title{
The effect of assist-as-needed support on metabolic cost during gait training of chronic stroke patients in LOPESII
}

\author{
Bertine. M. Fleerkotte, Jaap. H. Buurke, Edwin. H. F. van Asseldonk and Johan.S. Rietman
}

\begin{abstract}
Effectiveness of robotic gait training in rehabilitation of stroke patients remains inconclusive. A reason could be that the current robotic gait trainers do not initiate motor learning principles enough. To encourage active participation of the patient and therefore motor learning, assistas-needed (AAN) support strategies have been implemented in the robotic gait trainer LOPESII. Aim of the current study was to examine the effect of assist-as-needed support on metabolic cost.

Ten chronic stroke patients completed three 6-minute walking trials in LOPESII, with zero support, AAN-support for stiff knee gait and complete-support. Metabolic parameters were measured and compared between support conditions.

No significant differences in net metabolic power were observed between zero-support, AAN-support and complete support.

No evidence was found that AAN-support asks a higher metabolic cost of the participant.
\end{abstract}

\section{INTRODUCTION}

Robotic gait training has been developed as gait training for individuals with neurological impairments. The use of robotic gait trainers over conventional gait training is not proven yet. Comparison between robot-assisted gait training and therapist-assisted gait training show lower muscle activity [1$2]$ and energy cost $[1,3]$ indicating less active participation, whereas active participation is necessary for motor learning [4]. Encouraging active participation can be achieved by a more adaptive approach, such as lowering the guidance force (GF) or using an assist-as-needed (AAN) support [5]. Three studies $[3,6,7]$ looked at the effect of lowering the guidance force on metabolic cost. A lower guidance force seems to increase the metabolic cost in stroke patients, spinal cord injured patients and healthy subjects, but the effects are very small. With regard to the AAN approach only results on muscle activity were available, showing a higher muscle activity [8] comparing to a more fixed approach. Research on metabolic cost during robotic gait training with the AAN approach is not available. Therefore, this study will focus on the effect of AAN support on the metabolic cost during gait training of chronic stroke patients.

This study is financial supported by Pidon, Ministry of Economic Affairs.

B. M. Fleerkotte is working at Roessingh Research and Development, Enschede, The Netherlands (corresponding author to provide e-mail: b.fleerkotte@ rrd.nl).

J. H. Buurke is working at Roessingh Research and Development, Enschede, The Netherlands.

\section{METHODS}

\section{A. Participants}

A total number of thirteen stroke patients $(7$ men and 6 woman; mean age $61.0 \pm 13.4$ ) were recruited. Inclusion criteria were 1) hemiparesis as a result of stroke, 2) age above 18 years, 3 ) a stable medical condition, 4) a functional ambulation category (FAC) score of $\geq 3,5$ ) presence of stiff knee gait observed by experienced physical therapists and 6) a physical condition that allows for three times six minutes of walking in LOPESII.

\section{B. Equipment}

\section{Robotic gait trainer LOPESII}

With LOPESII the entire kinematic trajectories of the upper and lower legs can be supported. LOPESII also allows adjustment of support on various aspects of gait. The support provided by LOPESII is manually adjustable, varying between zero and $100 \%$ impedance. By adjusting the amount of support and supporting a specific aspect of gait, LOPESII has the ability to provide Assist-As-Needed (AAN) support.

\section{Cosmed}

Metabolic cost during walking sessions in LOPESII was measured using a portable metabolic unit $\left(\mathrm{K}_{4} \mathrm{~b}^{2}\right.$, Cosmed s.r.l., Rome, Italy).

\section{Protocol}

All measurements were performed in one session. Participants performed three walking trials with different levels of support; zero-support, AAN-support on foot clearance and complete-support. The order of trials was randomized. Each walking session consisted of six minutes of walking followed by a 10-minute rest period. In the complete-support condition, the general guidance force was increased till the same level of support as the amount of foot clearance in the AAN-support condition. A comfortable walking speed in LOPESII was selected for each participant individually. This comfortable walking speed was used for each support condition.

After each walking condition, the participant assessed his perceived physical effort on the 20-point Borg Rating of Perceived Exertion Scale.

E. H. F. van Asseldonk is working at University Twente, Enschede, The Netherlands.

J.S. Rietman is working at Roessingh Research and Development, Roessingh Rehabilitation Centre and University Twente, Enschede, The Netherlands. 


\section{Data Analysis}

For four gait parameters, the symmetry was calculated: step width, step length, stance time and swing time.

The metabolic cost was calculated using the measured rates of oxygen consumption and carbon dioxide production. For each trial, measured $\mathrm{V}^{\cdot} \mathrm{O}_{2}\left(\mathrm{ml} \mathrm{O}_{2} \mathrm{~s}^{-1}\right)$ and $\mathrm{V}^{\cdot} \mathrm{CO}_{2}(\mathrm{ml}$ $\mathrm{CO}_{2} \mathrm{~s}^{-1}$ ) was averaged over the last 30 -second interval of each support condition.

\section{E. Statistical Analysis}

The means of each participant of each of the three conditions was used for statistical analysis. If data proved to be normally distributed, a repeated measures ANOVA was performed to test for differences between conditions.

\section{RESULTS}

For ten out of thirteen patients in the study measures were analyzed. Three patients couldn't complete the walking session.

Individual gait speed ranged between subjects from 1.4 to $2.7 \mathrm{~km} / \mathrm{h}$ (mean $2.1 \pm 0.4 \mathrm{~km} / \mathrm{h}$ ). Amount of support ranged from 15 to $90 \%$ (mean $43 \pm 24 \%$ ). The maximal knee flexion angle deviated less from the reference maximal angle when support was provided. There was a significant decrease in peak flexion angle deviation in the AAN-support compared to the zero-support $(\mathrm{p}<0.05)$ and in the complete-support condition compared to zero-support $(\mathrm{p}<0.05)$.

\section{Metabolic Measures}

Figure 1 shows the mean and standard deviation of the net metabolic power and the individual data of the participants. The results show that there is a great variance between participants. Participants 5 and 7 show an increase in metabolic power at the AAN support compared to the complete support, However, the net metabolic power was not significantly different between support conditions, $\mathrm{F}(2$, 18) $=1.720, p>0.05$.

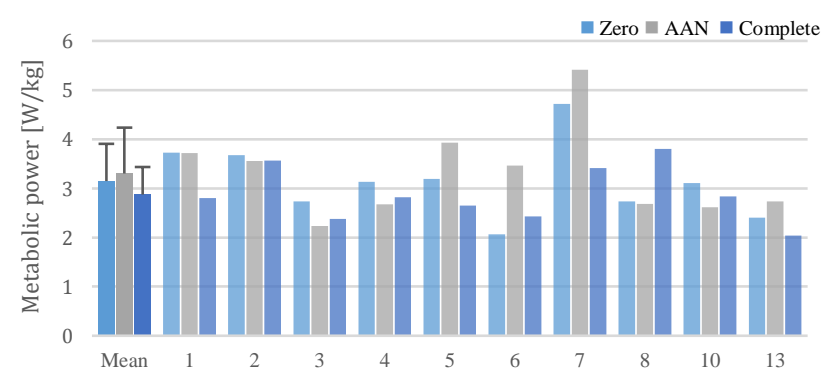

Fig. 1. Mean and standard deviation, and individual net metabolic power for the zero-support, AAN-support and complete support conditions.

\section{Borg Scores}

Results show that the provided support does not significantly affect the scores on the Borg rating of perceived exertion scale ( $p>0.05$ ). Mean score on the Borg scale for zero-support was $11.9 \pm 2.1$, for AAN-support 12.7 \pm 1.7 and for complete-support $12.3 \pm 3.1$.

\section{Discussion}

The aim of the present study was to examine the effect of assist-as-needed support on metabolic cost of chronic stroke patients walking in LOPES II. Metabolic cost was expected to be higher in the AAN-support condition, compared to complete-support. Although there was a trend towards lower metabolic cost in complete support, this trend did not reach significance. Other studies [6,7] also did not find a difference in metabolic cost after asking more activation by lowering the GF. No other study reported about the effect on metabolic cost using zero support, because this was not possible in the current gait trainers. Metabolic cost during walking seems to be dependent of many factors, like walking speed and step length asymmetry [9] and balance support [10]. A limitation in this study were the large differences between participants in gait speed and the use of walking aids.

\section{CONCLUSION}

This study shows that there is no evidence that assist-asneeded support leads to a higher metabolic cost than complete support by a robotic gait trainer.

\section{REFERENCES}

[1] J. F. Israel, D. D. Campbell, J. H. Kahn, T. G. Hornby. Metabolic costs and muscle activity patterns during robotic- and therapistassisted treadmill walking in individuals with incomplete spinal cord injury. Phys Ther. 2006: 86 (11); 1466-78.

[2] K. van Kammen, A. M. Booonstra, L. H. V. van der Woude, H. A. Reinders-Messelink, R. den Otter. Differences in muscle activity and temporal step parameters between Lokamat guided walking and treadmill walking in post-stroke hemiparetic patients and healthy walkers. J NeuroEng and Rehab. 2017: 14-32.

[3] M.P. van Nunen, K.H. Gerrits, A. de Haan, T.W. Janssen. Exercise intensity of robot-assisted walking versus overground walking in nonambulatory stroke patients. J Rehabil Res Dev. 2012; 49: 15371546.

[4] M. Lotze, C. Braun, N. Birbaumer, S. Anders, L. G. Cohen. Motor learning elicited by voluntary drive. Brain, 2003: 126 (4); 866-872.

[5] L. Marchal-Crespo, D. J. Reinkensmeyer. Review of control strategies for robotic movement training after neurologic injury. J NeuroEng and Rehab. 2009: 6 (20);

[6] C. Krewer, F. Muller, B. Husemann, S. Heller, J. Quintern, E. Koenig. The influence of different Lokomat walking conditions on the energy expenditure of hemiparetic patients and healthy subjects. Gait \& Posture. 2007: 26; 372-377.

[7] S. Y. Lee, E. Y. Han, B. R. Kim, M. H. Chun, Y. K. Lee. Can lowering the guidance force of robotic-assisted gait training induce a sufficient metabolic demand in subacute dependent ambulatory patients with stroke? Arch Phys Med and Rehab. 2017: 98; 695-700.

[8] A. Duschau-Wicke, A. Caprez, R. Riener. Patient-cooperative control increases active participation of individuals with SCI during robotaided gait training. J NeuroEng and Rehab. 2010: 7 (43)

[9] L. N. Awad, J. A. Palmer, R. T. Pohlig, S. A. Binder-Macleod, D. S. Reisman. Walking speed and step length asymmetry modify the energy cost of walking after stroke. Neurorehabil Neural Repair. 2015: 29(5); 416-423.

[10] T. Ijmker, H. Houdijk, C.J. Lamoth, A. V. Jarbandhan, D. Rijntjes, P. J. Beek, L. H. van der Woude. Effect of balance support on the energy cost of walking after stroke. Arch Phys Med and Rehab. 2013; 94 : 2255-61 